\title{
Information Recall Support for Elderly People in Hyper Aged Societies
}

\author{
Hsin-Hsi Chen \\ Department of Computer Science and Information \\ Engineering, National Taiwan University, Taiwan \\ MOST Joint Research Center for AI Technology and All \\ Vista Healthcare, Taiwan \\ hhchen@ntu.edu.tw
}

\author{
Manabu Okumura \\ Laboratory for Future Interdisciplinary Research \\ of Science and Technology \\ Institute of Innovative Research \\ Tokyo Institute of Technology, Japan \\ oku@pi.titech.ac.jp
}

\begin{abstract}
Memory loss, common seen in elderly people, affects their social interaction in the daily life very much. This 3-year international project jointly funded by Taiwan Ministry of Science and Technology (MOST) and Japan Science and Technology Agency (JST) investigates together the crucial issues behind the hyper aged societies. We aim at developing technologies and systems to provide information recall support for elderly people at the right time and at the right place.
\end{abstract}

\section{CCS CONCEPTS}

- Computing methodologies $\rightarrow$ Natural language processing • Applied computing $\rightarrow$ Law, social and behavioral sciences

\section{KEYWORDS}

Lifelogging; memory recall; personal big data

\section{INTRODUCTION}

Various kinds of problems arise in accompany with age increase. Memory loss is common seen in elderly people. Elderly people are much easier to forget things in the daily life. They may forget their operative skills for important tasks. They may forget the names of the exact entities in the conversation with other persons. This Taiwan-Japan joint project is aimed at developing related ICT technologies to provide information recall support for elderly people at the right time and at the right place.

\section{ACM Reference format:}

H.H. Chen, and M. Okumura. 2018. Information Recall Support for Elderly People in Hyper Aged Societies. In The 2018 Web Conference Companion (WWW 2018), April 23-27, 2018, Lyon, France, IW3C2, 2 pages. DOI: https://doi.org/10.1145/3184558.3186202

This paper is published under the Creative Commons Attribution-NonCommercialNoDerivs 4.0 International (CC BY 4.0) license. Authors reserve their rights to disseminate the work on their personal and corporate Web sites with the appropriate attribution.

WWW'18 Companion April 23-27, 2018, Lyon, France.

(c) 2018 IW3C2 (International World Wide Web Conference Committee), published under Creative Commons CC BY 4.0 License.

ACM ISBN 978-1-4503-5640-4/18/04.

DOI: https://doi.org/10.1145/3184558.3186202

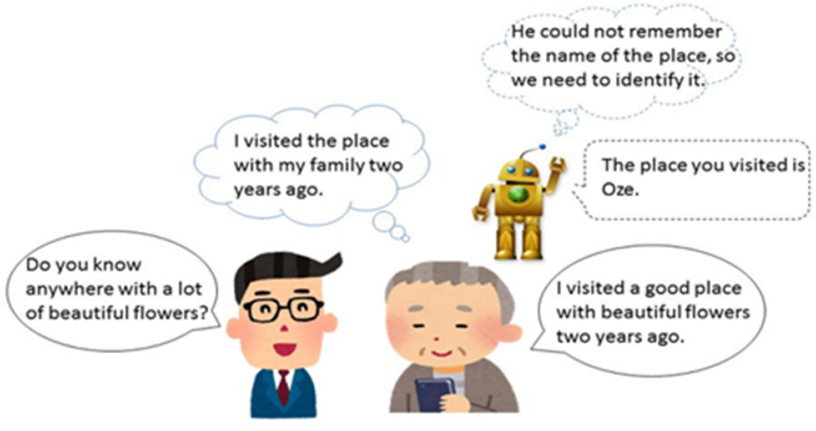

Figure 1: Usage scenario of information recall support.

One scenario of the use of information recall service is shown as follows. We have encountered many situations where we need to recall past experiences; for example, we may want to recall who was at a party we attended, or where we went in 2000 . When elderly people forget some specific named entities during talking, the information recall service reminds the elderly people of the specific entities they would like to mention. For example, consider the situation in Figure 1. When the user's friend asked him if he knows anywhere with a lot of beautiful flowers, he tried to recommend the place where he visited with his family two years ago. However, he could not remember the name of the place and just said he visited a good place with beautiful flowers two years ago. In this situation, the service tries to identify the name of the place by searching the knowledge base related to his experience using the information 'he visited a place with beautiful flowers two years ago'. That will decrease the barrier between elderly people and other persons in their conversation and resolve dilution of human relationship.

This international project (April 1st 2017 March 31st 2020) was funded jointly by MOST (Taiwan) and JST (Japan) under the cooperative research topic "ICT for Accessibility and Support of Older People”. The Taiwan-based and Japan-based PIs are Professors HsinHsi Chen and Manabu Okumura, and the project volumes for both sides are NTD4.74 million and JPY17.55 million, respectively. The total budget of the three years is equal to USD311,950 or EUR267,675. The project web site please refer to http://nlg.csie.ntu.edu.tw/mostjst-joint-project/. 


\section{RESEARCH ITEMS}

In the information recall support, knowledge base construction and knowledge base retrieval are two sides of a same coin. A knowledge base serves as an auxiliary memory of elderly people, which keeps all the entities, entity properties, and entity relations (events) related to the elderly people past experience. It is a personal (local) knowledge base rather than a global knowledge base like DBpedia. On the one hand, in the knowledge base construction, an information recall support system first collects multimedia data of real-world life experience of individuals, such as their documents, their photos, and their videos. It then organizes the collected data with a focus on identifying and annotating or indexing events.

On the other hand, in the knowledge base retrieval, the information recall support system should know when elderly people needs the service and provides answers based on their private memory. Different from traditional dialogue management systems, which deal with human-computer interaction, the information recall support system also provides the service even during human-human conversations, as illustrated in Figure 1. Therefore, the system should retrieve the answer from the user's knowledge base without any input from her by taking into account the information in a sequence of her utterances as a query.

The information recall support system provides two modes to help elder persons shown in Figure 2. In the reactive mode (Figure 2(a)), it looks like a personal question-answering agent. It provides answers for questions from an elder person. Two parties will be involved. In the proactive mode (Figure 2(b)), it intervenes the human conversation as a partner to remind the elderly people the missing information. At least three parties are involved.

In the proactive mode, we focus on the system intervention in the conversations of elderly people with other persons. The issues to be tackled include: (1) detect the turn of the elderly people in the conversation; (2) capture the need of information recall service from elderly people; (3) tell the types of information recall for the services; (4) find the answers for the services if the intention is clear; (5) guide the elderly to recall memory if intention is not clear.

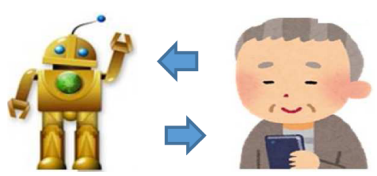

(a) Reactive Mode

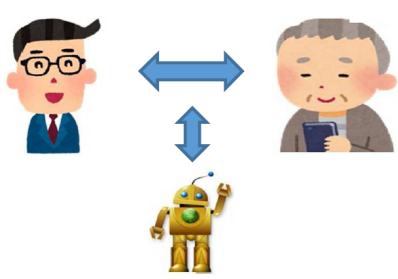

(b) Proactive Mode
Figure 2: Modes of information recall supports systems.

\section{LIFE LOGGING}

Both global and local knowledge bases are useful for information recall support service. Global knowledge base, which keeps world knowledge, is available from public domain. Local knowledge base, which keeps personal knowledge, is the target to be established. The raw data comes from daily life.
In the first year of this project, we design a logging mechanism to collect personal data from heterogeneous sources. That include the social network of a specific people and his/her social events. Currently, life diary written in tweets and traveling diary written in blogs are collected as the experimental datasets. The content includes text and images. Besides, we extract the basic named entities from the personal data, find their relationships, and organize events in temporal order. People, affairs, time, places, and things surrounding the specific people are five major targets. Moreover, we design a representation scheme to keep the knowledge extracted by information extraction technologies. In traditional knowledge graph, each node represents an entity and each link between entities denotes their relationship. To extend the facet-based representation to capture the temporal order is one of research issues.

\section{RELATED WORK}

Estrin [2] talked about the potential use of the digital traces we generate daily. Gurrin, Smeaton, and Doherty [4] called life logging as personal big data, and proposed some potential applications of the lifelogging data. Gurrin et al. [3] presented the first lifelog dataset and used it in NTCIR retrieval evaluation. Althoff et al. [1] proposed an idea of TimeMachine, and generated relations between entities based on knowledge base. Li et al. [5] extracted some major life events such as marriage and graduation from tweets.

The above researches show the trend of lifelogging. This international project is different from those researches in several aspects. We will extract open events in daily life from logging. Moreover, we focus on the proactive information recall in human conversation. When to intervene the conversation and how to solve the disfluencies of the conversation based on the personal knowledge base are challenging issues.

\section{CONCLUSIONS}

This paper presents an international research project to cope with memory loss problem. Ideas of information recall services are introduced. The overall pictures include life logging, conversion of personal big data to knowledge base, and reactive and proactive information recalls. Rapid aging of population in the world is a global issue. Besides Japan and Taiwan, most countries in the world will face the same problem. We will share the experiences from this international project and deal with the global challenges together with other researchers.

\section{REFERENCES}

[1] Tim Althoff, Xin Luna Dong, Kevin Murphy, Safa Alai, Van Dang, Wei Zhang. 2015. TimeMachine: Timeline Generation for Knowledge-Base Entities. In Proceedings of KDD'15, 19-28.

[2] Deborah Estrin. 2015. What Could We Do with Access to Our Small Data? http://www.www2015.it/keynote-speakers/

[3] Cathal Gurrin, Hideo Joho, Frank Hopfgartner, Liting Zhou and Rami Albatal. 2016. NTCIR Lifelog: The First Test Collection for Lifelog Research, In Proceedings of SIGIR 2016, 705-708.

[4] Cathal Gurrin, Alan F. Smeaton and Aiden R. Doherty. 2014. LifeLogging: Personal Big Data. Foundations and Trends in Information Retrieval. 8(1): $1-107$.

[5] Jiwei Li, Alan Ritter, Claire Cardie and Eduard Hovy. 2014. Major Life Event Extraction from Twitter based on Congratulations/Condolences Speech Acts. In Proceeding of 2014 EMNLP, 1997-2007. 